\title{
MEDICAL IMAGING AND PROCESSING METHODS FOR CARDIAC FLOW RECONSTRUCTION
}

\author{
KELVIN K. L. WONG* \\ Center for Biomedical Engineering \\ and School of Electrical 83 Electronics Engineering \\ University of Adelaide, SA 5005, Australia \\ kwong@eleceng.adelaide.edu.au \\ RICHARD M. KELSO \\ School of Mechanical Engineering \\ University of Adelaide, SA 5005, Australia \\ S. G. WORTHLEY and P. SANDERS \\ Cardiovascular Research Centre \\ Royal Adelaide Hospital and School of Medicine \\ University of Adelaide, SA 5005, Australia \\ J. MAZUMDAR and D. ABBOTT \\ Center for Biomedical Engineering \\ and School of Electrical \& Electronics Engineering \\ University of Adelaide, SA 5005, Australia
}

Received 20 December 2008

Accepted 20 January 2009

Intra-cardiac blood flow imaging and visualization is challenging due to the processes involved in generating velocity fields of flow within specific chambers of interest. Visual analysis of cardiac flow or wall deformation is crucial for an accurate examination of the heart.

Cardiac chamber boundary encapsulation is one of the key implementations for region definition. To provide intelligible results describing flow within the human heart, cardiac chamber segmentation is a pre-requisite so that fluid motion information can be presented within a region of interest defined by the chamber boundary. A technique that is used to establish contouring along the cardiac wall is described mathematically. This article also sets the practical foundation for flow vector synthesis and visualization in the cardiac discipline. We have outlined conceptual development and the construction of flow field based on a three-dimensional Cartesian grid that can give a greater insight into the blood dynamics within the heart.

We developed a framework that is able to present both anatomical as well as flow information by overlaying velocity fields over medical images and displaying them in cinemode. By addressing most of the methods involved from the programming perspective,

\footnotetext{
${ }^{*}$ Corresponding author.
} 
procedural execution and memory efficiency have been considered. Our implemented system can be used to examine abnormal blood motion behaviour or discover flow phenomena in normal or defective hearts.

Keywords: Flow visualization; velocity; segmentation; active contour; magnetic resonance imaging; motion tracking.

\section{Introduction}

An accurate vision of blood flow patterns within cardiovascular structures can assist in the discovery of flow phenomena in a human heart and is useful for investigation of cardiac abnormalities. Therefore, cardiac visualization dominates the level of data understanding as inaccurate presentation may obscure the crucial details of measurement. We have designed and constructed an interactive visualization system to achieve good visual display that is suitable for three-dimensional blood flows. Such a framework possesses interactivity so that the state of flow over a cardiac cycle can be deciphered easily and effectively.

In this paper, we have also examined the active contour algorithm that is used for semi-automatic segmentation of heart chambers for region segregation and flow analysis. In reality, based on cardiac magnetic resonance images (MRI), it is often difficult to achieve full automation for such application due to the poor definition of cardiac wall boundary and artefacts within the blood pool. Therefore, we implemented a semi-automatic segmentation algorithm when active contouring fails to define internal boundaries of the heart chamber effectively. Flow velocity grids within segmented regions are developed for analysis at a later stage. Useful visualization tools that can be applied to present flow optimally are described.

Three-dimensional flow reconstructions can be performed using successive planar vector velocity maps. Such a flow field can be constructed based on $x$-, $y$ - and $z$-components of each velocity vector in space. The visualization of the flow can be supported with tools such as streamline tracing. In the absence of the third normal velocity component, due to the limitation of a velocimetry system to determine the rate of movement of blood normal to the plane of scan, we can construct this missing component by superimposing the in-plane components of two planes that are orthogonal to the current plane. Therefore, we can extend two-dimensional flow analysis to a three-dimensional one by taking each set of the three planar vectors from the $x-y, y-z$ and $x-z$ planes respectively and constructing them into a single resultant flow in space iteratively using vector addition. It may be worthwhile mentioning that although such technology has been implemented before, ${ }^{1,2}$ the programming aspect of reconstruction has not been discussed and most applications are not within the cardiac discipline.

We have described a translation system that is implemented to assist in reconstructing parallel planes of medical images of the heart. Scanned vector maps can be combined using our visualization framework to present flow with suggested strategies related to optimal processing of its display. Furthermore, various sections in 
this chapter outline user-program interacting mechanisms and procedures for controlling operations of the visualization from a software development perspective. The conceptual framework that is presented can also be utilized in future work related to flow visualization and fluid analysis in other fields.

\section{Two-Dimensional Segmentation of Cardiac Structures}

\subsection{Introduction to active contour technique}

A segmentation approach known as active contouring that can be used to define the region of interest for flow analysis within the heart. Active contours exist in different forms, such as parametric and geometric deformable models. ${ }^{3}$ The active contour model can be implemented using a snake contour, which is an energy-minimizing spline guided by external constraint forces and influenced by image forces that dynamically pull it toward features such as lines and edges in digital images. ${ }^{4}$ These are computational curves that deform to localize object shapes represented on these images. In this section, we will focus on describing and utilizing the parametric active contour. It is worthwhile mentioning that mechanism of active contouring can be extended to perform three-dimensional segmentation. ${ }^{5}$

\subsection{Energy minimization of parametric snake}

Active contouring is applied onto gray-scale intensity MR images for segmentation of cardiac chambers. A region of interest (ROI) can be established by the expansion of a preliminary active contour that is positioned within the examined chamber.

Movement of cardiac tissues is effectively excluded by performing contour segmentation on the cardiac walls. The cardiac wall is segmented by placement of a two-dimensional contour that forms a computationally elastic wall within the cardiac chamber. Migration of contour nodes from their origins onto the intense wall region is executed based on an energy minimization algorithm. ${ }^{6,7}$

Active contouring computes a more accurate contour-line description iteratively by describing the contour as an energy function $E_{\text {contour. It receives information }}$ from the preceding contour line and applies energy balancing based on the internal and external energies of this line denoted by $E_{\text {int }}$ and $E_{\text {ext }}$ respectively to redefine the contour representation. The best fitted contour is one that corresponds to the minimum of this energy:

$$
E_{\text {contour }}=\int E_{\text {int }}+\int E_{\text {ext }} .
$$

The initial curve can be anywhere in the image, and interior contours are automatically detected. In the event of poor segmentation due to over-expansion of the elastic contour, the internal wall of the chamber is manually traced to adapt more to the shape of the region of interest before expansion takes place. Because of the semiautomatic nature of segmentation, contour tracing algorithm set in our program is 
not the best choice for application. We emphasize that the techniques outlined in this paper may not be the most efficient and effective methods of implementation at the current stage; however, the principles outlined here can be improved for better performance subsequently.

\subsection{Implementation of parametric snake model}

In the previous section, we describe qualitatively the concept of the snake model and its deformation. Now, we will discuss the mathematical aspects of the model which effectively uses the Kass snake algorithm. ${ }^{4}$

An active contour can be defined as a parametric curve $v(s)=[x(s) y(s)], s \in$ $[0,1]$, with each configuration component associated with a finite energy. We define the first and second order of $v(s)$ as $v^{\prime}$ and $v^{\prime \prime}$ respectively. Expansion of Eq. (1) gives

$$
E_{\text {contour }}=\int E_{\text {elastic }}+\int E_{\text {bending }}+\int E_{\text {ext }}
$$

where

$$
\begin{aligned}
\int E_{\text {elastic }} & =\frac{1}{2} \int_{0}^{1} \alpha(s)\left|v^{\prime}(s)\right|^{2} d s \\
\int E_{\text {bending }} & =\frac{1}{2} \int_{0}^{1} \beta(s)\left|v^{\prime \prime}(s)\right|^{2} d s,
\end{aligned}
$$

and

$$
\int E_{\text {bending }}=\int_{0}^{1} P[v(s)] d s .
$$

The mechanical properties of the contour are controlled by parameters, $\alpha$ and $\beta$ which determine the snake's tension and rigidity respectively to provide elasticity for snake deformation. Here, $P$ is the potential associated with the external forces and proportional to the spatial intensity gradient of the image. The external energy $E_{\text {ext }}$ is designed to enable migration of snake nodes towards the step edges of grayscale image which is an intensity matrix $I(v)$,

$$
P[v(s)]=-|\nabla I[v(s)]|^{2} .
$$

The energy equation can be minimized using the Euler Lagrange Differential Equation. Using calculus of variations, it can be shown that the minimum energy configuration corresponds to the equation

$$
\alpha v^{\prime \prime}(s)-\beta v^{\prime \prime \prime \prime}(s)-\nabla P[v(s)]=0 .
$$

In this formulation, each term acts as a force applied to the contour. The whole configuration can be viewed as an equation to balance forces:

$$
F_{\text {int }}+F_{\text {ext }}=0 \text {. }
$$

The internal force $F_{\text {int }}=\alpha v^{\prime \prime}(s)-\beta v^{\prime \prime \prime \prime}(s)$ sets the rigidity and elasticity of the snake contour while the external potential force $F_{\text {ext }}=-\nabla P[v(s)]$ attracts the 
contour towards the edges of an image object. The snake migrates towards the local minima of the potential $P$ which is equivalent to the local maxima of the spatial intensity gradient.

\subsection{Segmentation of cardiac atria using active contouring}

Most medical image segmentation of the heart requires expert human intervention to achieve accurate and consistent segregation of the region from the rest of the other cardiovascular structures. In some cases, active contouring may outperform other segmentation techniques such as region growing ${ }^{8,9}$ especially when a user-inserted boundary representing the atrial chamber in the image is required to prevent overflow of pixels into the connecting vessels of an atrium. However, the control of energy pertaining to the snake may be difficult and full automation may not be realizable in a robust and reliable medical image segmentation tool.

Based on Fig. 1, we demonstrate the use of contour snakes for interactive segmentation of cardiac chambers. Initial plantation of a closed loop contour can be within the chamber of interest. Expansion for a balloon of elemental nodes can be activated semi-automatically by migration of the nodes towards the chamber wall with interactive user control. When there is poor definition of myocardium continuity around an atrium, active contouring of the endocardium may fail and deform out of the geometrical boundary due to weak energy levels to constraint over expansion. Especially for cardiac magnetic resonance imaging of atria, the scanned heart chamber image shows poor definition of the atrial wall and automated contouring using computer algorithms is difficult. In the event of such failures, we take over the automation by supervising the planting and growth of snake contour nodes within the chamber manually. This allows us to obtain a more accurate cardiac chamber contouring and segmentation.

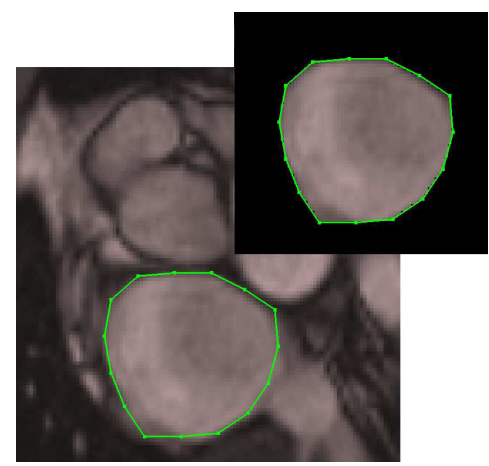

(a) Right atrium segmentation

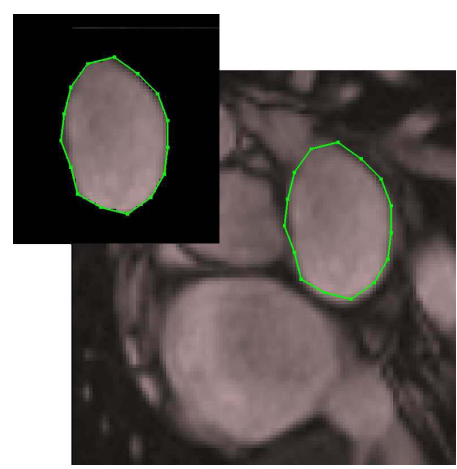

(b) Left atrium segmentation

Fig. 1. Segmentation of atria based on active contours. Active contouring is applied onto MR images to perform boundary definition of cardiac chambers which can be used for segmentation of the chamber of interest. Active contours in the form of snakes utilise the intensity contrast of the cardiac wall and blood pool within to define the boundary region of the right and left atria. 


\section{Two-Dimensional Flow Image Reconstruction}

The flow region defined within a region of interest can be displayed using reconstruction of velocity flow vectors over the segmented area only. To aid visualization, anatomic information such as the magnetic resonance images of the scan can be superimposed onto the flow field such that outside the area of interest, the details of the cardiac wall and other cardiovascular chambers are shown. Such a configuration can help in a more concise visualization of flow in the chamber of examination and the anatomical structures around it.

The system demonstrated in Fig. 2 shows the stages involved in reconstructing a series of temporal flow images that can be played back like a movie clip to aid flow visualization in the heart. The development of two-dimensional flow images can sometimes be sufficiently informative to explain flow behavior in the cardiac chambers. It is also easier to quantify two-dimensional flows statistically as compared to three-dimensional ones.

Flow visualization tools based on colour ribbons, streamlines and vector plots can be applied depending on the user preference and the nature of the fluid flow. ${ }^{11}$ For cardiac examination, it may be useful to determine the speed of blood in cardiovascular systems. Therefore, color streamlines like the ones shown in Fig. 3 are commonly used. We emphasize again that cardiac segmentation is performed to

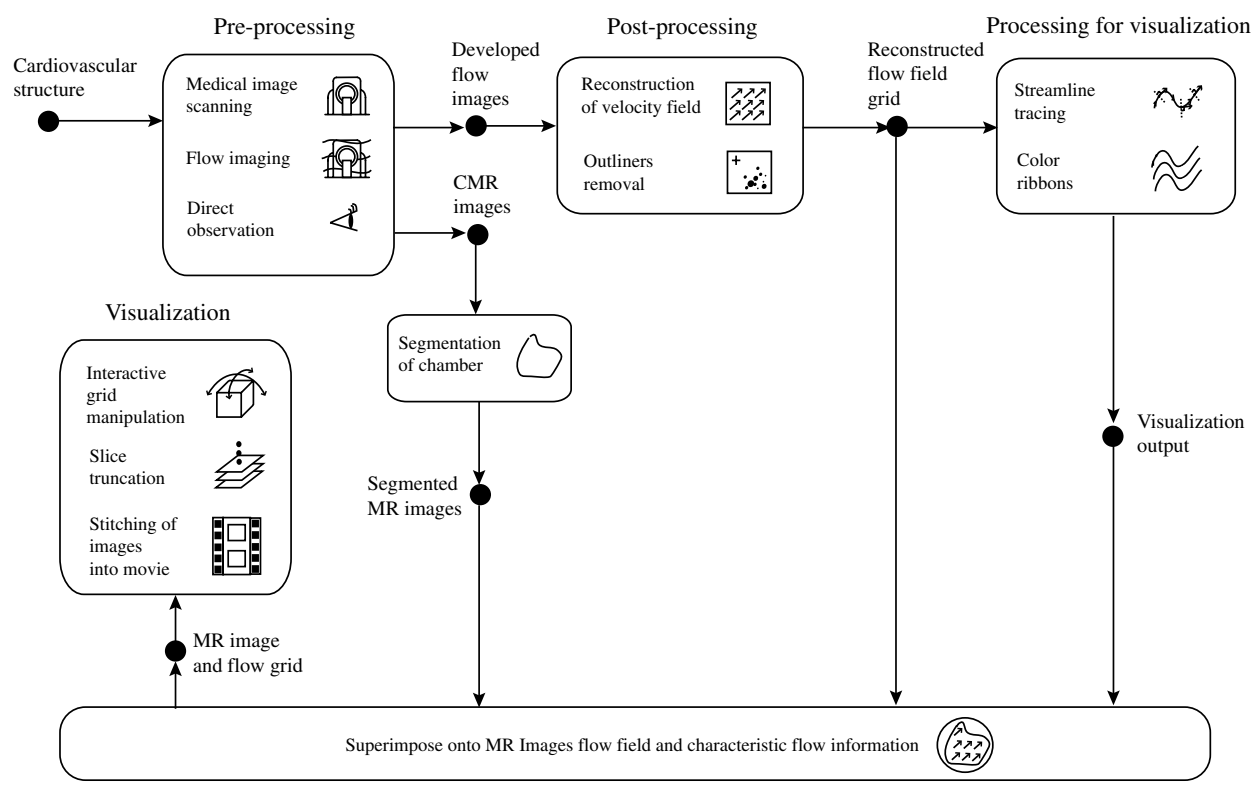

Fig. 2. A cardiac velocity visualization system. Visualization of blood motion is provided by its velocity flow field within the segmented chamber of interest. To achieve this final flow image, various stages such as segmentation, flow field reconstruction, and superimposition of images must be carried out. In addition to the production of flow images, streamline tracing and interactive image slice manipulation can also be performed. 


\section{CARDIAC FLOW}

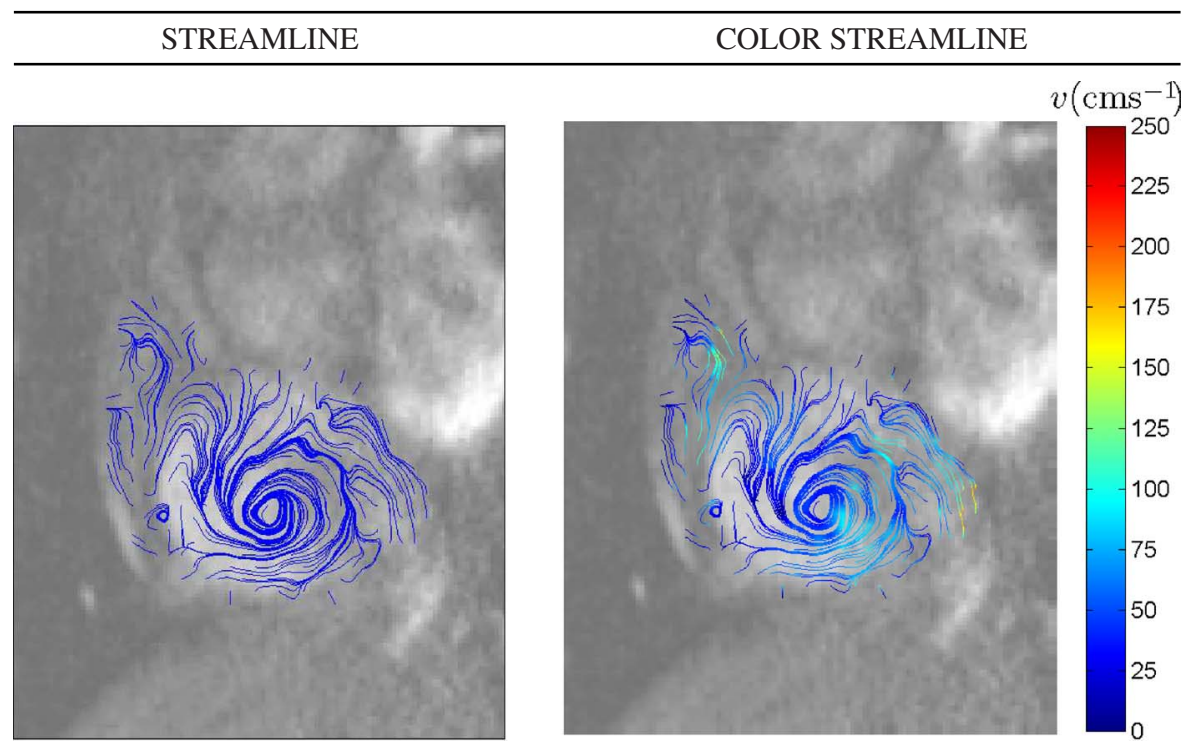

Fig. 3. Streamline visualization of flow in a normal heart. Streamline plots (with and without colors) are displayed for velocity map based on phase contrast magnetic resonance imaging. Using streamline tracing, it is possible to indicate swirling within a heart chamber. The color intensity of the streamlines can be used to describe how fast the blood is rotating within an enclosed space by referencing the velocity scale. Streamline flow visualization is a useful technique to examine cardiac flow phenomena in the heart.

limit the display of streamlines within the chamber of interest. The segmented set of streamlines is overlayed onto the medical image to highlight the location of blood flow examination and enables the identification of the chamber easily. In our example, the magnetic resonance image produced as a background canvas, illustrates the anatomical details of the heart while providing flow information at the same time. Multiple flow images can be stitched together to create a movie playback of the changing flow patterns and anatomical deformations. This allows users to view the temporal information related to blood fluid and cardiovascular structural interaction effectively.

\subsection{Phase contrast VENC MRI velocimetry}

We perform flow field scans using phase contrast magnetic resonance imaging of a healthy right atrium. The phase contrast MRI technology is clinically attractive because it is able to provide quantitative information on blood flow without the need for contrast agent to be introduced into the human body. ${ }^{12-15}$

The velocity-encoded MR imaging was performed using a Siemens Avanto, 1.5 Tesla, model-syngo MRB15 scanner with Numaris-4, Series No: 26406 software. Cine-MR imaging was performed using one slice in short axis views through the 
atria. All images were acquired with retrospective gating and 25 time frame indices (from $n_{t}=1$ to 25 ) for a single slice.

Phase contrast magnetic resonance imaging is used to scan the normal subject. Acquisition parameters include: $\mathrm{TR}=47.1 \mathrm{~ms}, \mathrm{TE}=1.6 \mathrm{~ms}, \mathrm{FOV}=298 \times 340 \mathrm{~mm}^{2}$ at matrix of $(134 \times 256)$ pixels. We focus our examination on atrial flow visualization in this experiment. This provides a useful way of viewing the entire development of the blood flow, instantaneously, to be able to obtain an understanding of vortex characteristics in the right atrium.

The MR imaging parameters for phase contrast velocity encoding protocols are chosen for the optimal flow field generation within the right atrium. We configure the tracking system so that the optimal tracking can be effected. The in-plane and through-plane resolution of the scans are determined by the pixel spacing at $1.54 \mathrm{~mm} /$ pixel and slice interval of $6 \mathrm{~mm}$ respectively.

We present some images based on this MRI protocol in Fig. 4. The phase contrast images are graphical representations of the velocity components $(x$ - and

\section{PHASE CONTRAST MRI}

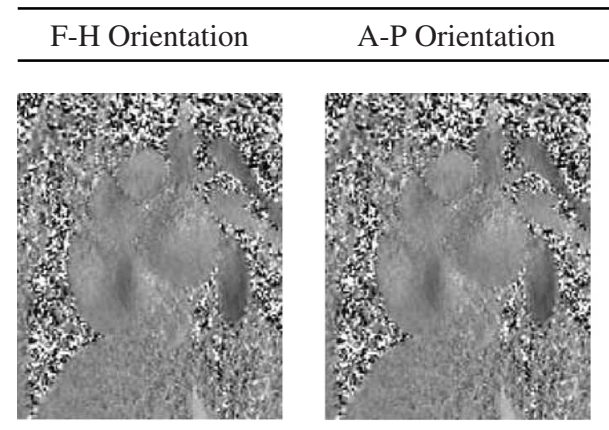

(i) $n_{t}=10$
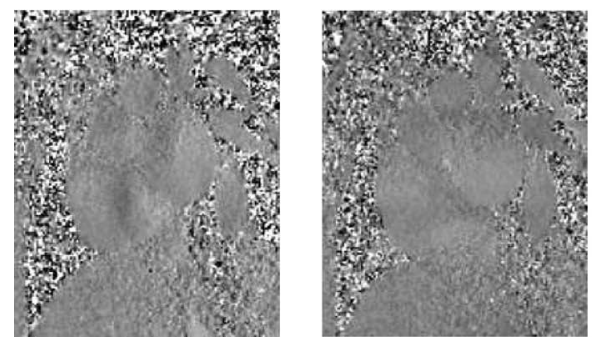

(iii) $n_{t}=12$

F-H Orientation A-P Orientation

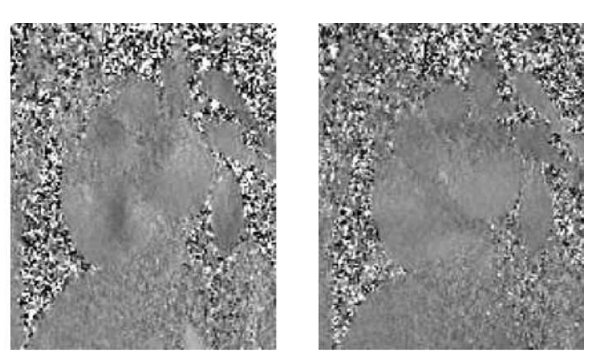

(ii) $n_{t}=11$
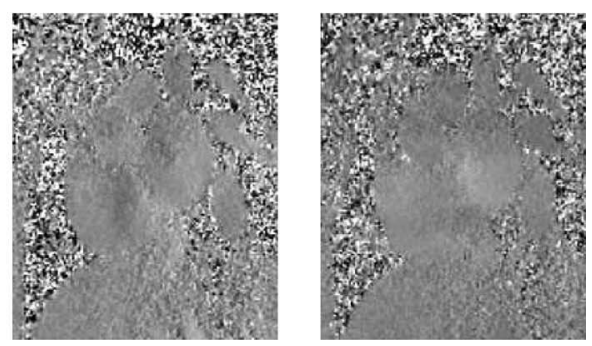

(iv) $n_{t}=13$

Fig. 4. Phase contrast images of cardiac chamber. Short axis scans pertaining to time frame indices $n_{t}=[10,11,12,13]$ out of 25 frames in a cardiac cycle is presented. Scans based on two orientations, namely the Foot-Head (FH) and the Anterior-Posterior (AP) are taken. The intensity of the pixels in the image indicates the magnitude of the velocity component in the specified orientation. Combining two orthogonal velocity-encoded image maps can produce a two-dimensional velocity flow field. 


\section{PHASE CONTRAST MRI VELOCITY FIELD}

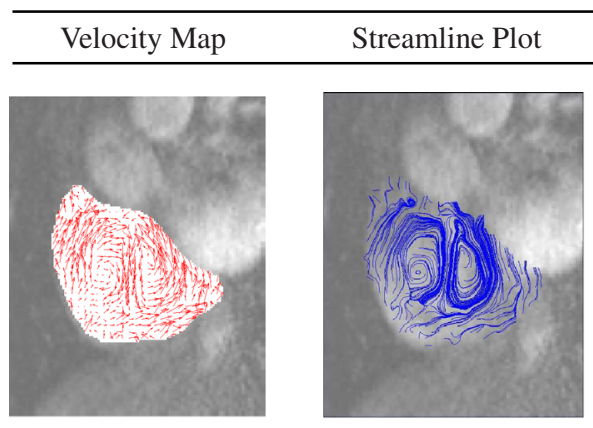

(i) $n_{t}=10$

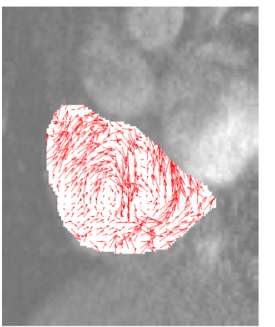

(iii) $n_{t}=12$

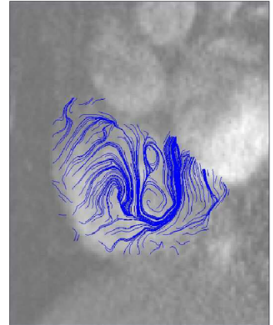

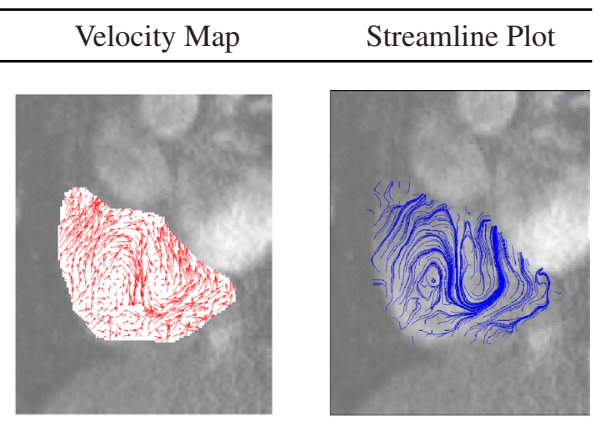

(ii) $n_{t}=11$
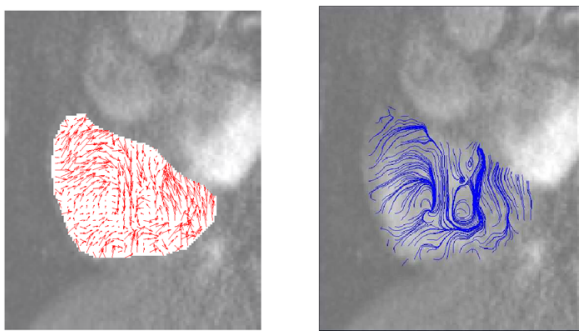

(iv) $n_{t}=13$

Fig. 5. Velocity field of cardiac chamber. The velocity fields for time frame indices $n_{t}=$ $[10,11,12,13]$ out of 25 frames in a cardiac cycle are created by combining the phase contrast images based on the $x$ - and $y$-directions. The fields are then presented using vector plots made up of multiple red arrows distributed within the region of interest. The flow patterns of the blood may be traced by streamlines (represented in blue) which gives an indication of the direction of flow within user-specified regions of interest.

$y$-directions) maps. The figure shows the Foot-Head $(\mathrm{F}-\mathrm{H})$ and Anterior-Posterior (A-P) orientation scans.

Combining the two velocity maps based on the in-plane $x$ - and $y$-directions results in the velocity field of blood as shown below in Fig. 5. Phase contrast MRI can be extended to three-dimensions as well by combining an additional orientation, the Left-Right ( $\mathrm{L}-\mathrm{R})$ image scans to obtain the through plane velocity component map. Then, having the $z$-velocity component in addition to the in-plane components will be possible.

As can be observed, phase contrast MRI encodes velocity information within the output images and we are able to decipher these data to produce velocity field maps for analysis of flow patterns within the cardiac chambers. The phase contrast framework enables the flow imaging of cardiovascular system non-invasively and with good reliability. This technology is well-established in the medical imaging industry and will be able to serve as a gold standard flow imaging protocol for validation of new methodologies that will be developed. 


\subsection{MR fluid motion tracking}

Other cardiac velocity reconstruction framework exists, of which one recent development is MR fluid motion tracking, ${ }^{10}$ that can offer high speed velocity field generation at the expense of accuracy. Fluid motion tracking is a computational method of estimating blood motion based on cardiac magnetic resonance images (MRI). We apply the term MR fluid particularly to a bio-fluid medium, such as blood, which experiences magnetic resonance (MR). Motion tracking refers to estimation of this fluid movement and generation of the flow field. We develop a tracking system that performs motion tracking of registered MR signals from blood in the heart.

A series of MR images are taken temporally as the fluid is in motion. The velocity of dynamic fluid can be quantified in real time by computing the shift of intensities within the quantised regions of every prior and post image numerically. A velocity flow field can be constructed using a graphical plot as such and other fluid dynamics properties can be derived from the velocity flow measurement. From the results, the characteristics of the fluid flow can be analyzed using these properties.

We can computationally determine the movement of fluid in a vessel based on motion estimation of the contrasting MR-signals. The motion of localized turbulence is influenced by the general flow globally. Motion estimation using multiresolution optical flow technique is able to track the movement of the flow at various resolutions and resolve them to produce a global flow field in two dimensions. Therefore, we termed this approach MR fluid motion estimation, as it is able to compute motion of MR imaged fluid.

Application of flow based on the use of motion estimation algorithm allows us to produce flow vectors over the region of defined analysis. This technique makes use of images from two subsequent phases to predict the flow field. Typically, cine MRI scanning results in a sequence of $N$ phases. Post-processing of the data from $(N-1)$ pairs of images gives a series of flow field displays for evaluation and analysis.

We have performed cardiac flow assessment of a pathological heart to provide an example of the MR fluid motion field of a patient who is diagnozed with atrial septal defect. Limited by the absence of the velocity-encoding (VENC) protocol during the MRI scanning of this patient, only steady-state free precession (SSFP) MR images are obtained to assess the septal defect by examining the discontinuity along the myocardium. The strategy of attaining a stronger assessment of the defect is by utilising the same SSFP MR images for both anatomical and flow evaluation. Figure 6 shows the derived velocity field from intensity motion tracking for both pre- and post-atrial septal occlusion at a sample time frame index out of 25 frames in one cardiac cycle.

Our set of results illustrates the potential clinical interest to analyze local blood motion behavior or to discover flow phenomena based on pathological conditions. For example, medical experts will be able to provide a study of a defective heart and compare it against the normal or the post-operation case. It should also have the capability to provide discussion on the difference that can be captured by this 


\section{VECTOR PLOT}

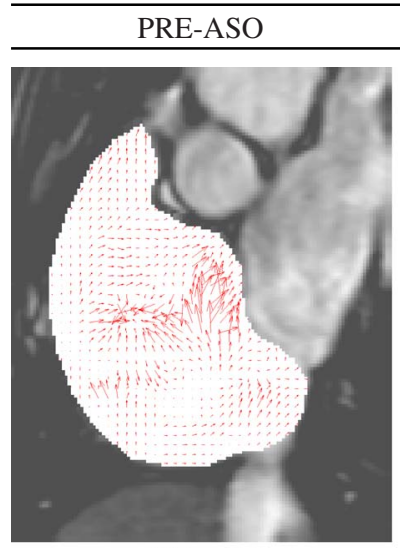

(i) Pre-ASO

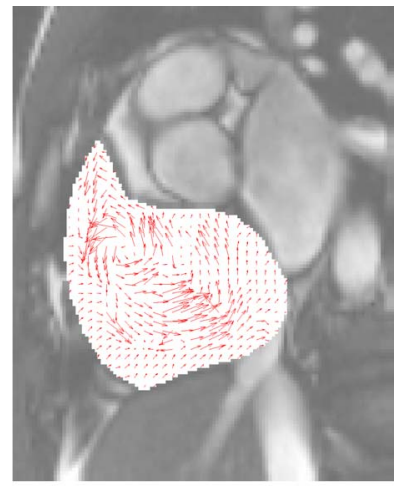

(ii) Post-ASO

Fig. 6. MR fluid motion field of right atrial flow pre- and post-ASO. One slice that passes through the septal defect has been choosen for flow analysis here. The size of the pre-ASO atrium is shown to be larger than that of the post-ASO one. It can be deduced from these scans that there is an imbalance of pressure maintained by both atria. In this case, pressure is increased in the right atrium due to the left to right shunting of blood which causes the blood circulation in the heart to lose efficiency.

system methodology, with the aim of flow grid reconstruction that requires short processing time and lower memory, for an effective and efficient diagnosis.

\section{Three-Dimensional Flow Image Reconstruction}

We describe a technique of combining three sets of planar velocity images pertaining to the axial, sagittal and coronal cardiac orientations into a three-dimensional flow field grid. This presents a method for higher dimensional reconstruction of flow if the imaging system is incapable of scanning the normal velocity component of the planar flow section. An example of an imaging system limited by through-plane flow scanning is digital particle image velocimetry. ${ }^{16,17}$ Unlike phase contrast magnetic resonance imaging, the implemented framework requires a longer processing time for flow grid reconstruction due to the extensive memory and computations required.

\subsection{Cartesian grid for image and flow display}

In general, we are able to produce vector fields in three orientations if flow images from three sets of planes orthogonal to each other are scanned throughout the heart. Typically, we define the planes as $x-y, y-z$ and $x-z$ in the axial, sagittal and coronal orientations of the flow volume respectively. If every planar slice at an orientation has a two-dimensional in-plane flow grid, then a resultant flow grid from the $X, Y$ and $Z$ flow grids can be reconstructed in three-dimensions. A schematic illustration 


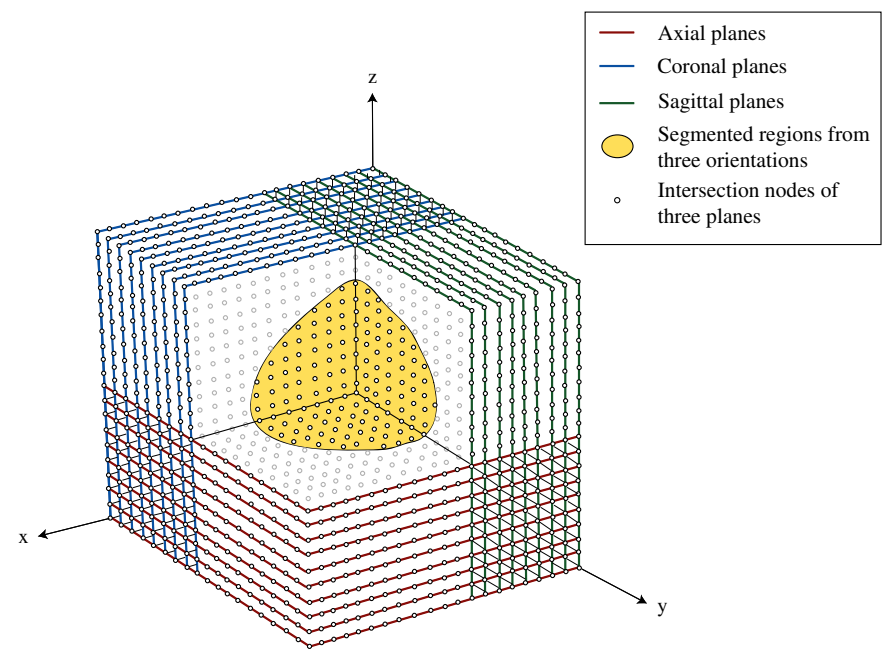

Fig. 7. Three-dimensional image grid reconstruction. Reconstruction of image grids based on the Cartesian framework can be performed. The nodes representing point of intersection of the three planes in the $x, y$ and $z$ orientations can be displayed. Each of these acts as points for flow construction by vector addition of the flow features associated to the node. The number of nodes will depend on the number of planes within a volume space.

of the image grid over the flow volume is shown in Fig. 7. Flow vectors within a volume of fluid can be constructed based on the image grid framework which we schematically demonstrate in Fig. 8.

A segmentation grid is imposed onto the data image construction. Any node of plane intersection and the resultant flow vector associated with it that is outside the region of segmentation will not be displayed. In this figure, a section of the axial, sagittal and coronal slices are hidden for a dissection of the flow volume. The nodes presented spatially in the volume demonstrate how a group of nodes spatially distributed in a three-dimensional space can be volumetrically segmented by applying two-dimensional segmentation for all of the orthogonal planes.

The Cartesian configuration of a field grid can be utilized for a three-dimensional construction of the flow; however, from the perspective of visualization, it does not help in providing much insight into the flow patterns. Therefore, it is definitely of interest to view flow in-plane from a selected orientation and via a single slice at each time of examination. Nevertheless, coupling the display of a three-dimensional grid of flow vectors with user-defined control of the grid in orientation and distance from a reference visual perspective can allow good visual control.

From the programming perspective, the images are stored in a five-dimensional matrix whereby the orientation $p$, spatial coordinates $x, y$ and $z$, and time frame index $n_{t}$ form each of the dimensions. Counts of $P$ orientations, with spatial sizes $I, J$ and $K$, and a total number of time frames $T$ contributes to $P \times I \times J \times K \times$ $T$ number of matrix elements. Figure 9 illustrates the image dimensions from a graphical perspective. 


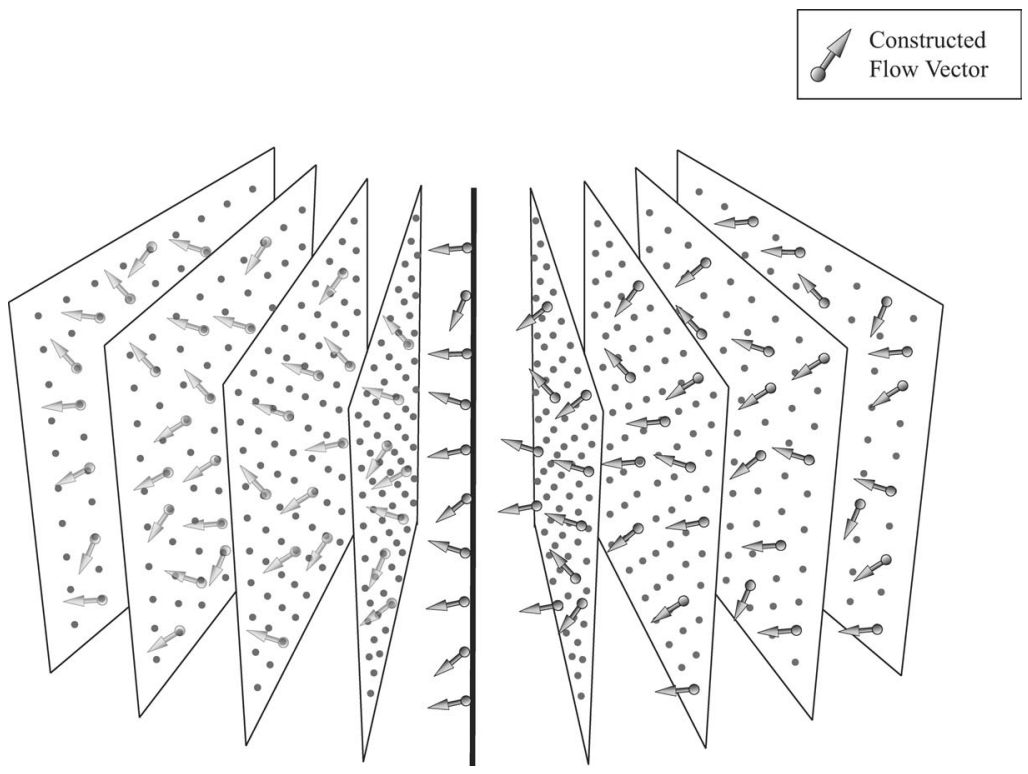

Fig. 8. Reconstruction of flow using vectors from three orientations. A three-dimensional grid can be constructed from the axial, sagittal and coronal planes. The vectors at the point of intersection of the three planes are added to produce the resultant flow at every node. Flow outside of the segmented region will not be computed. So only the vectors within the region of encapsulation by the contour will be displayed and attributed to the vector addition. Therefore, we will have a display of three-dimensional vectors within the segmented heart chamber that is constructed from three orientations of two-dimensional in-plane vector grids.
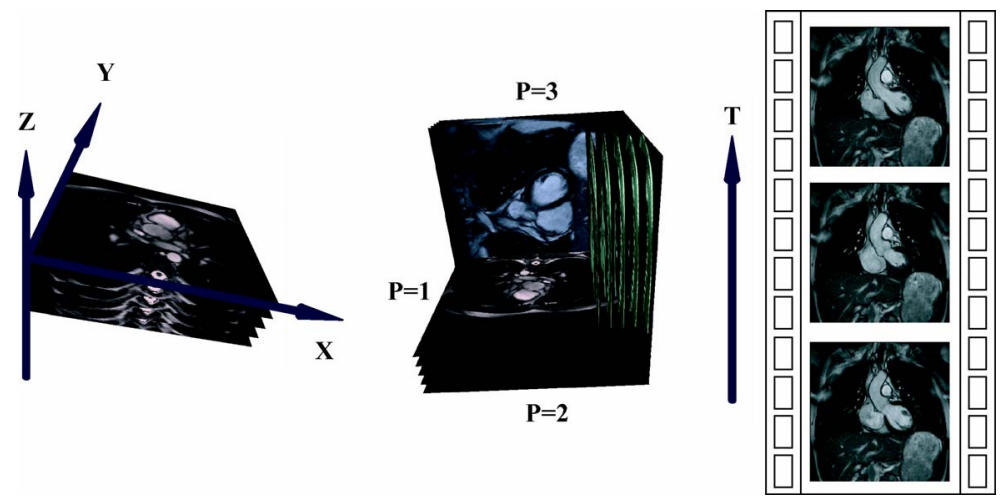

Fig. 9. Construction of image matrix based on five dimensions. The matrix that is used for processing and display of images has five dimensions. These dimensions are based on arrays labelled as $X, Y, Z, P$, and $T$. Because of the huge storage matrix required for allocation of data, good memory management is required in the software application. For example, storage memory during running of program is always released after flow grids are processed. Another strategy in good memory conservation is to resize the matrix when images are cropped or slices are truncated. 


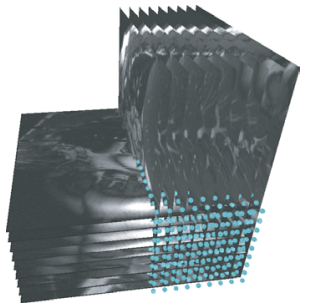

(a) Two sets of planes

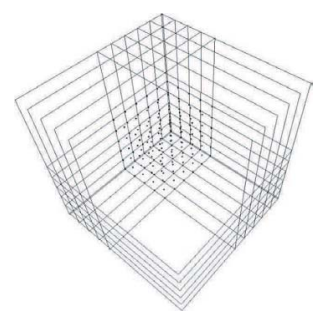

(c) Truncated transparent planes

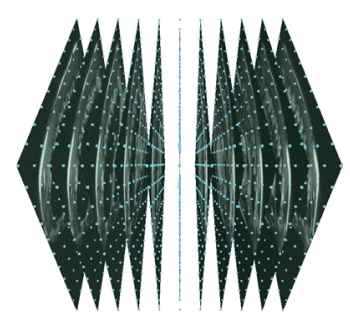

(b) One set of planes

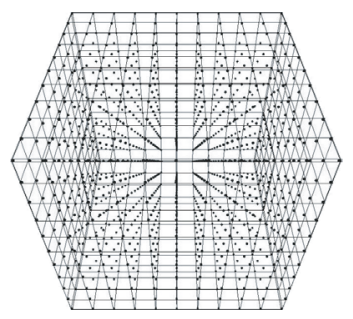

(d) Full set of transparent planes

Fig. 10. Intersection nodes of a three-dimensional grid. Reconstruction of MRI slices using three orientations, and with spheres anchored at the points of intersection of the slices in a threedimensional space. The two diagrams (a) and (b) in the figure show the graphical outputs by our program while (c) and (d) illustrate the construction of nodes in space schematically.

Therefore, for three-dimensional analysis of flow, the MRI slices from the axial, sagittal and coronal scans can be computationally constructed into a stack grid as shown in Fig. 10. On the display platform, the points of intersection of three orthogonal planes are represented by spherical points at junctions of intersection. As an example and for good demonstration purposes, the axial planes are hidden and inter-slice distance has been reduced to reveal these points.

In (a), the coronal planes and part of the axial and sagittal planes have been hidden to allow visualization of the anchored spheres at junctions of plane intersections. Based on (b), the number of junctions in a three-dimensional grid of planes is limited by the number and spacing of the slices for axial, sagittal and coronal orientations. Therefore, a dense image grid is required for accurate three-dimensional computational measurements of flow. However, for demonstration purposes, we display a stack of appropriately spaced images forming a grid structure that is able to reveal the intersectional junctions visually. By setting the planes in schematic outlines, we are able to explain this graphically. Part (c) shows that sections of orthogonal planes are set in hidden mode for clear illustration of the plane intersection junctions in this schematic setup. A full set of intersecting planes are shown in (d) and the transparency property of the image slice can reveal the number of junctions. 


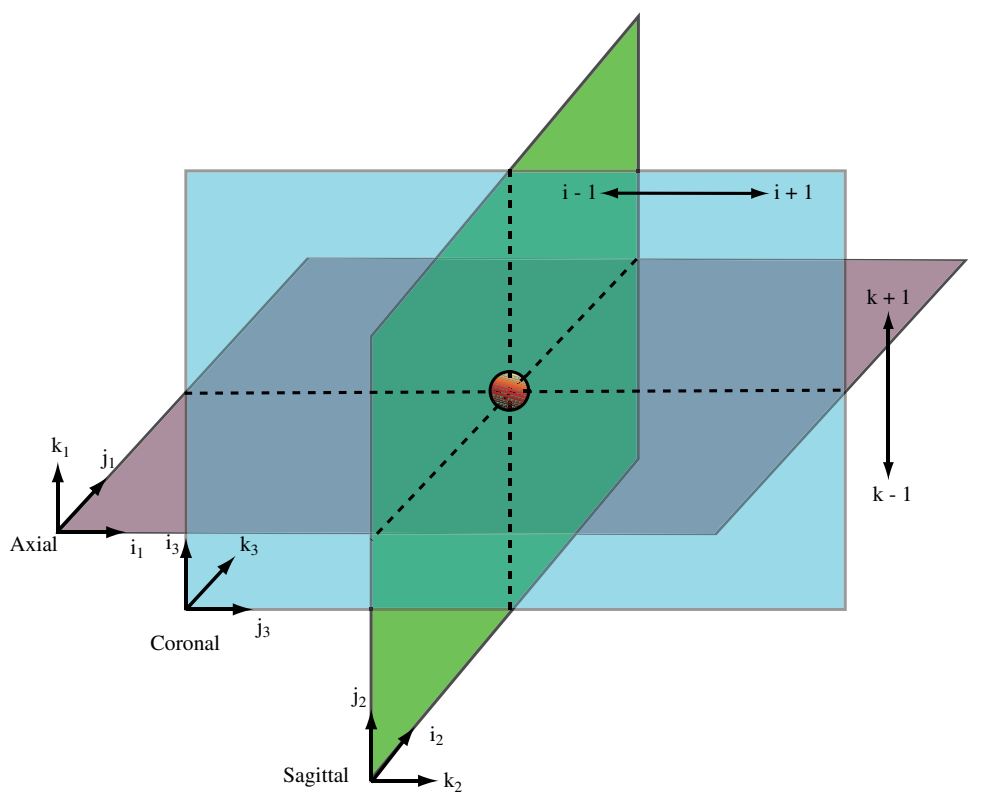

Fig. 11. Geometrical representation of plane intersection. Reconstruction of flow using vectors from three orientations, namely, the $x-y, y-z$ and $x-z$ planes. Each node that is associated with an orientation has three orthogonal flow vector components in the $i, j$ and $k$ directions. The intersection from each of the other two orientations will contribute a set of two vectors corresponding to each plane. In total, there are six vector components that are associated with a node based on intersection from three planes. Therefore, the attribute of each node is a single flow vector whose $i, j$ and $k$ components are based on the addition of these vectors.

\subsection{Computation of flow grid}

We can perform vector addition through addition of the vectors from $x-y, y-z$ and $x-z$ planes. This gives us a three-dimensional flow of designated magnitude and direction at the node of intersection of the three planes as seen in Fig. 11.

Two-dimensional vectors in-plane at arbitrary coordinates are denoted by

$$
\begin{array}{r}
{\left[v_{i}^{\text {Axial }}\left(x_{1}, y_{1}, z_{1}, n_{t}\right), v_{j}^{\text {Axial }}\left(x_{1}, y_{1}, z_{1}, n_{t}\right)\right],} \\
{\left[v_{i}^{\text {Sagittal }}\left(x_{2}, y_{2}, z_{2}, n_{t}\right), v_{j}^{\text {Sagittal }}\left(x_{2}, y_{2}, z_{2}, n_{t}\right)\right],}
\end{array}
$$

and

$$
\left[v_{i}^{\text {Coronal }}\left(x_{3}, y_{3}, z_{3}, n_{t}\right), v_{j}^{\text {Coronal }}\left(x_{3}, y_{3}, z_{3}, n_{t}\right)\right]
$$

which pertain to the axial, sagittal and coronal orientations respectively. Note that $x_{p}, y_{p}, z_{p}$ for $p=1,2$ and 3 measures the spatial position of the point of intersection of the three planes. This intersection node is referenced from an origin at $[0,0,0]$. This flow grid corresponds to images pertaining to time frame indices $n_{t}=1$ to $M$. The equations for adding $i, j$ and $k$ directional flow vectors at every point 
of intersection of the three flow planes give us the orthogonal components $V_{i}, V_{j}$ and $V_{k}$ of the resultant flow as a function of position $[x, y, z]$ and time frame index $n_{t}$ is:

$$
\begin{gathered}
V_{i}\left(x, y, z, n_{t}\right)=v_{i}^{\text {Axial }}\left(x_{1}, y_{1}, z_{1}, n_{t}\right)+v_{j}^{\text {Coronal }}\left(x_{3}, y_{3}, z_{3}, n_{t}\right), \\
V_{j}\left(x, y, z, n_{t}\right)=v_{i}^{\text {Sagittal }}\left(x_{2}, y_{2}, z_{2}, n_{t}\right)+v_{j}^{\text {Axial }}\left(x_{1}, y_{1}, z_{1}, n_{t}\right),
\end{gathered}
$$

and

$$
V_{k}\left(x, y, z, n_{t}\right)=v_{i}^{\text {Coronal }}\left(x_{3}, y_{3}, z_{3}, n_{t}\right)+v_{j}^{\text {Sagittal }}\left(x_{2}, y_{2}, z_{2}, n_{t}\right),
$$

given that for $1 \leq x \leq I, 1 \leq y \leq J$ and $1 \leq z \leq K$. We also note that

$$
x=x_{1}=z_{2}=y_{3}, \quad y=y_{1}=x_{2}=z_{3}, \quad z=z_{1}=y_{2}=x_{3} .
$$

Root-mean-square of the vector gives the resultant vector magnitude $V_{R}$ as

$$
\left|V_{R}\right|=\sqrt{\left(V_{i}\right)^{2}+\left(V_{j}\right)^{2}+\left(V_{k}\right)^{2}} .
$$

Figure 12 shows the display of arbitrary flow vectors based on intersection of flow image slices in three orientations. For every intersection point, a resultant vector based on the three orthogonal velocity components can be computed. The analysis of three-dimensional flows is limited by the poor inter-slice resolution and lack of contour segmentation. For illustration of our method, we have used a reduced number of anchor spheres for demonstration of an effective visualization. Flow fields that are used in the generation of these images are arbitrary and has been graphically presented based on the programming strategies that have been discussed.

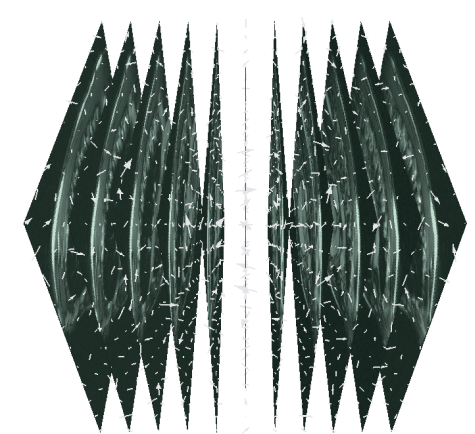

(a) Plane and intersection nodes

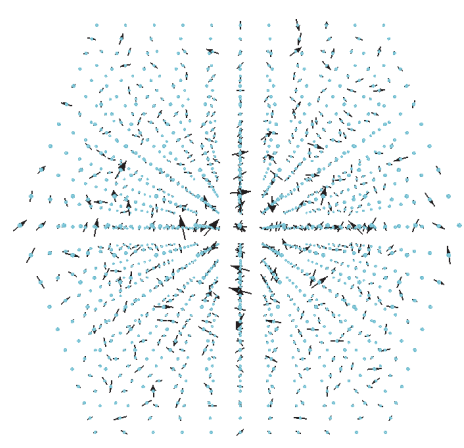

(b) Intersection nodes only

Fig. 12. Measured flow vectors in a three-dimensional space through image planes. Based on part (a), we illustrate the augmentation of MR images on three-dimensional grid of the intersected measurements. This enhances a user's view by overlaying graphical and anatomical information onto it. The flow directions can be represented using arrows and its speed correspond to the magnitude of velocity vectors in space. From (b), we see that the configuration of flow vector display in three spatial dimensions is based on the number and density of intersection junctions. 


\section{System Limitations}

The methodological limitation of this work is discussed based on the MRI protocol capability as well as the image registration and scan quality. This section mentions the type of computational techniques that should be applied and also discusses system limitations associated with it.

\subsection{Availability of VENC MRI protocol}

The mathematical framework derived in this work is applicable dependent on the availability of the scan protocol and the dimension of the flow analysis. For velocityencoded imaging modalities, the post-processing computational expense is low considering that most of the processing time is shifted to the physical measurement of localized blood velocity components. Unlike MR fluid motion tracking, system calibration and image registration of the motion-sensitized MR-signal measured during successive scans, compensates the reconstruction of velocity grid using intensity flow estimation that is more time consuming. However, comparing the time taken to encode velocities during phase contrast MRI scanning, the overall speed of velocity grid development is much higher.

While MR fluid motion tracking may be efficient based on planar velocity grid construction, the construction of a single three-dimensional grid based on successive planes of flow grids may be computationally inferior to imaging techniques that are able to measure through-plane fluid velocities (an additional velocity component that is perpendicular to in-plane ones). Reconstruction using scans from the latter technique is able to produce such a flow grid with three sets of velocity-encoded images pertaining to the orthogonal orientations quickly and with low computational cost. However, there may be limitation of an imaging protocol to achieve the through-plane velocity measurement. Emerging technologies such as magnetic resonance fluid motion tracking which can only predict in-plane velocity fields may find our mathematical framework applicable when developing three-dimensional flow grids.

\subsection{Effect of scan resolution and image quality on SSFP MRI}

A magnetic resonance image is reconstructed using every pixel, where gray-scale intensity (brightness) is proportional to the amplitude of MR signal that corresponds to volume element of specific dimensions in the slice. Therefore, the low turbulent regions will have a higher signal intensity emitted by the region of voxels to contribute brighter pixels in the image. On the contrary, higher turbulence results in de-phasing of spins within the voxels and tends to reduce the signal intensity that shows up as darker pixels.

The accuracy of tracking signal motions is dependent on the quality and resolution of the signals represented in magnetic resonance images. A larger area of measurement scope will give a higher quality of flow field production since a greater 
information set of signal pixels can be utilized for motion estimation. Ideally, the series of images that will be useful for fluid motion tracking should have a high signal-to-noise ratio, high spatial resolution, superior soft tissue contrast and no ghosting artifacts; which however, comes at the expense of imaging time. ${ }^{18}$ Noise in MR images is the result of thermally driven Brownian motion of electrons present in the conducting body or even within the receiving coil itself. ${ }^{19}$ Spatial resolution increases at the cost of reducing signal-to-noise ratio.

\subsection{Parameters affecting MR fluid motion tracking}

We suggest that for signal emitting nuclei motion that are represented by intensity pixels on MR images, the application of multi-resolution optical flow scheme predicts fluid motion based on grey-level constancy assumption (optical flow constraint). In magnetic resonance imaging terminology, field of view (FOV) is defined as the size of the two- or three-dimensional spatial encoding region of the image, and is usually defined in units of $\mathrm{mm}$. MR imaging spatial resolution ( $\mathrm{mm} / \mathrm{pixel})$ is defined as the ratio of field of view $(\mathrm{mm})$ to image matrix (pixels). If the imaging resolution improves, more details can be captured within the scanned image and a higher collection of MR signals can be registered per unit area of the magnetic resonance imaged flow. Spatial resolution defines the capability of resolving the turbulent flow details. MR signals from a voxel are collectively averaged and result in loss of micro-turbulence details smaller than the voxel of nuclear information. Therefore, the dimension of the voxel determines the smallest scale of turbulence in fluid that can be registered onto image. Due to aliasing and attenuation, this scale of turbulence may be several voxels wide.

\subsection{Insufficient quantification by velocity information}

Flow visualization by parametric motion field alone is often insufficient in characterising fluid rotation. Note that although such velocity information is limited in a concise description of the flow, the use of additional quantities such as strain rates can help us to gain more insight into the fluid motion. Vorticity derivation can be used to denote rotational fluid flow about a point. A vorticity field of the cardiac region of interest can be developed to provide useful information for potential clinical applications involving cardiac flow interpretation and analysis. However, it is not the scope of this paper to present vorticity visualization and the techniques of a more concise analysis using this differential quantity.

\section{Conclusion}

Cardiac flow imaging and visualization involves multiple unique tools that can be combined to produce a system specially targeted for presentation of blood flow within the heart most effectively. The framework is designed to produce flow field 
displays with low processing cost and with maximum speed. We also describe in this paper, the development of framework for performing segmentation using active contouring on two-dimensional images. The flow field of the MR images can be presented with a more focused analysis if active contouring is performed onto the chamber boundary and any flow vectors outside of the established boundary will be hidden, thereby, presenting a clear overview of the blood pool dynamics within the segmented chamber.

Vector addition based on slices from three planes of orientation allows the development of a volumetric grid of flow vectors. Although a three-dimensional flow grid may not be of much utility in terms of analysis at the current stage of research, it may still be of interest to develop interactive displays of such information for flow visualization purposes. Future applications may evolve out of such a system and analysis of three-dimensional flows may be of better accuracy sometimes. But in cardiac flow imagings that we have outlined in this paper, we have simplified flow analysis to that of a two-dimensional one just to establish the foundation of this development and to gain preliminary insight into the volumetric flow. The development technique, that is based on the interpolation of multiple orthogonal planar flow planes, can also be applied onto non-cardiac related flow construction. For example, three-dimensional flow grid reconstruction may be based on particle image velocimetry that develops two-dimensional flow grid in any plane.

The primary aims of this paper are specifically directed at presenting a conceptual framework for post-processing magnetic resonance images and flow field scans with specific application in flow visualization of blood within cardiac chambers of the heart. This presents capability in processing the flow field for characterisation and subsequent analysis of interesting flow phenomenon or diagnosis of cardiac problems such as atrial fibrillations and septal defect. The programming aspects of this methodology are also outlined which can give an indication of the computational expense involved. The practical aspects of this technique is highly recommended for use in cardiac flow visualizations that involve both fluid and structural information such as blood and anatomical region of interest for analysis.

\section{References}

1. Alsberg E, Trahey G, Bohs LN, Friemel B, Nightingale K, Walker W, Threedimensional flow visualization via reconstruction from successive two-dimensional vector velocity maps, Proceedings of the 16th Annual International Conference of the IEEE 1:598-599, 1994.

2. Bohs LN, Friemel BH, Kisslo J, Harfe DT, Nightingale KR, Trahey GE, Threedimensional flow images by reconstruction from two-dimensional vector velocity maps, J Am Soc Echocardiogr 8(6):915-923, 1995.

3. $\mathrm{Xu} \mathrm{C}$, Prince JL, Snakes, shapes, and gradient vector flow, IEEE Transactions on Image Processing 7(3):359-369, 1998.

4. Kass M, Witkin A, Terzopoulos D, Snakes: Active contour models, International Journal of Computer Vision 1(4):321-331, 1988. 
5. Ahlberg J, Active contours in three dimensions, Ph.D. dissertation, Linköping University, 1996.

6. Cohen LD, On active contour models and balloons, Computer Vision, Graphics, and Image Processing 53(2):211-218, 1991.

7. Jasjit SS, Computer vision, pattern recognition and image processing in left ventricle segmentation: The last 50 years, Pattern Anal Appl 3(3):209-242, 2000.

8. Hao X, Bruce C, Pislaru C, Greenleaf JF, A novel region growing method for segmenting ultrasound images, Ultrasonics Symposium, 2000 IEEE 2:1717-1720, 2000.

9. Gonzalez RC, Woods RE, Digital Image Processing, 2nd edition. Prentice-Hall, Inc., New Jersey, USA, 2002.

10. Wong KKL, Kelso RM, Worthley SG, Sanders P, Mazumdar J, Abbott D, Theory and Validation of magnetic resonance fluid motion estimation using intensity flow data, PLoS ONE 4(3):e4747, 2009.

11. Lodha SK, Pang A, Sheehan RE, Wittenbrink CM, UFLOW: Visualizing uncertainty in fluid flow, IEEE Visualization '96, 249-254, 1996.

12. Powell AJ, Maier SE, Chung T, Geva T, Phase-velocity cine magnetic resonance imaging measurement of pulsatile blood flow in children and young adults: In vitro and in vivo validation, Pediatr Cardiol 21:104-110, 2000.

13. Herold V, Mörchel P, Faber C, Rommel E, Haase A, Jakob PM, In vivo quantitative three-dimensional motion mapping of the murine myocardium with PC-MRI at 17.6 T, Magnetic Resonance in Medicine 55:1058-1064, 2006.

14. Yamashita S, Isoda H, Hirano M, Takeda H, Inagawa S, Takehara Y, Alley MT, Markl M, Pelc NJ, Sakahara H, Visualization of hemodynamics in intracranial arteries using time-resolved three-dimensional phase-contrast MRI, J Magn Reson Imaging 25:473$478,2007$.

15. Mark M, Harloff A, Bley TA, Zaitsev M, Jung B, Weigang E, Langer M, Hennig J, Frydrychowicz A, Time-resolved 3D MR velocity mapping at 3T: Improved navigatorgated assessment of vascular anatomy and blood flow, Journal of Magnetic Resonance Imaging 25:824-831, 2007.

16. Alahyari A, Longmire E, Particle image velocimetry in a variable density flow: Application to a dynamically evolving microburst, Experiments in Fluids 17:434-440, 1994.

17. Raffel M, Willert C, Kompenhans J, Particle Image Velocimetry, eds. Adrian RJ, Gharib M, Wolfgang M, Rockwell D, Whitelaw JH, Springer-Verlag, Berlin Heidelberg, Germany, 1998.

18. Basic Principles of MR Imaging, Philips Medical Systems, 1st edn., 1984.

19. Redpath TW, Signal-to-noise ratio in MRI, The British Journal of Radiology 71:704$707,1998$. 